\title{
The DAWGPAWS pipeline for the annotation of genes and transposable elements in plant genomes James C Estill ${ }^{* 1}$ and Jeffrey L Bennetzen ${ }^{2}$
}

Address: ${ }^{1}$ Department of Plant Biology, The University of Georgia, Athens, Georgia 30602-7271, USA and ${ }^{2}$ Department of Genetics, The University of Georgia, Athens, Georgia 30602-7223, USA

Email: James C Estill* - JamesEstill@gmail.com; Jeffrey L Bennetzen - maize@uga.edu

* Corresponding author

Published: 19 June 2009

Plant Methods 2009, 5:8 doi:10.1 |86/1746-48|I-5-8

This article is available from: http://www.plantmethods.com/content/5/I/8

(c) 2009 Estill and Bennetzen; licensee BioMed Central Ltd.

This is an Open Access article distributed under the terms of the Creative Commons Attribution License (http://creativecommons.org/licenses/by/2.0), which permits unrestricted use, distribution, and reproduction in any medium, provided the original work is properly cited.

\begin{abstract}
Background: High quality annotation of the genes and transposable elements in complex genomes requires a human-curated integration of multiple sources of computational evidence. These evidences include results from a diversity of $a b$ initio prediction programs as well as homologybased searches. Most of these programs operate on a single contiguous sequence at a time, and the results are generated in a diverse array of readable formats that must be translated to a standardized file format. These translated results must then be concatenated into a single source, and then presented in an integrated form for human curation.

Results: We have designed, implemented, and assessed a Perl-based workflow named DAWGPAWS for the generation of computational results for human curation of the genes and transposable elements in plant genomes. The use of DAWGPAWS was found to accelerate annotation of 80-200 kb wheat DNA inserts in bacterial artificial chromosome (BAC) vectors by approximately twenty-fold and to also significantly improve the quality of the annotation in terms of completeness and accuracy.

Conclusion: The DAWGPAWS genome annotation pipeline fills an important need in the annotation of plant genomes by generating computational evidences in a high throughput manner, translating these results to a common file format, and facilitating the human curation of these computational results. We have verified the value of DAWGPAWS by using this pipeline to annotate the genes and transposable elements in 220 BAC insertions from the hexaploid wheat genome (Triticum aestivum L.). DAWGPAWS can be applied to annotation efforts in other plant genomes with minor modifications of program-specific configuration files, and the modular design of the workflow facilitates integration into existing pipelines.
\end{abstract}

\section{Background}

Genomic sequence assemblies are rapidly being published for a great number of species $[1,2]$. The sequence data used to produce genome assemblies are being generated at ever-increasing rates for reduced costs [3], indicating that the genomes of many more plant species will be de novo sequenced in coming years. The relative value of these sequencing efforts is a direct function of the accuracy of the annotation of the resultant sequence assemblies. Genome annotation seeks to delineate the sequence features that occur on the genome, thereby permitting definition of the biological processes responsible for these 
features [4]. In plants, the sequence characteristics that are most critical to our interpretation of gene function and genome evolution include both genes and transposable elements (TEs) $[5,6]$.

Identification of the genes that have been uncovered in assembled genome sequence data can utilize evidence from both $a b$ initio gene annotation programs as well as sequence similarity searches against databases of previously identified proteins and expressed RNA $[4,7,8]$. The $a b$ initio gene finding programs derive full gene models from DNA sequence data based solely on knowledge of the sequence features associated with protein coding domains. Sequence alignments can refine the exon-intron boundaries of these models and provide evidence that computationally predicted genes are actually transcribed in vivo. Existing software can automatically synthesize these data to derive combined evidence gene models $[9,10]$.

While this combination of $a b$ initio and homology-based approaches have been used to accurately annotate genes in a number of eukaryotic genomes, plant genome annotation efforts cannot focus solely on the annotation of genes due to the risk of conflating genes with transposable elements [11]. Many TEs contain open reading frames (ORFs) that generate the proteins required for TE transposition. The $a b$ initio gene annotation programs will often annotate these TE ORFs as genes. Since most TE genes are expressed and represented in cDNA libraries, homologybased searches will indicate that these ORFs are transcribed and they thus may be considered legitimate gene predictions. Simply removing the high-copy-number candidate genes does not alleviate this problem because some true gene families are highly abundant while not all transposable elements are highly repetitive [12]. These erroneous gene annotations are especially problematic in plant genomes where transposable elements make up the majority of sequenced genome space. Since these false positive gene predictions cannot be mitigated by gene prediction methods alone, plant genome annotation must directly annotate TEs in order to remove them from the gene candidate list.

Similar to the prediction of genes, accurate identification of the TEs in genomic sequence data combines homologybased searches and $a b$ initio results [13-15]. Tools for $a b$ initio transposable element discovery can exploit the fact that many families of TEs occur in high copy number within a host genome [16-18], or they can utilize diagnostic structural features such as tandem inverted repeats (TIRs) or long terminal repeats (LTRs) that delineate an individual TE insertion [19-21]. Homology-based searches of transposable elements are facilitated by specialized tools [22-25] that make use of databases of previ- ously identified TEs [26-29] or leverage repetitive data from the sequenced genome [30-32].

The gold standard of genome annotation is the integration and curation of multiple computational results by a knowledgeable biologist [11]. This approach has been advocated for the structural annotation of genes [4,11], as well as transposable elements [33]. A limitation of the manually-curated multiple-evidences approach is that the process requires the combination of computational results from a disparate set of independent annotation programs. The output of this software has been designed to maximize readability by humans and not to facilitate integration of results across programs. Furthermore, these tools are often designed to work on a single contiguous sequence (contig) at a time, while many annotation efforts require the generation of computational results for thousands of assembled contigs. Computational workflow suites that seek to aid in plant genome annotation must therefore overcome these limitations while facilitating the human interpretation of the computational results contributing to a biological annotation.

Here, we introduce an annotation suite that allows for computational evidences to be generated in an automated fashion, integrates the results from multiple programs and facilitates the human curation of these computational results. This suite was designed to assist a Distributed Annotation Working Group (DAWG) approach for a Pipeline to Annotate Wheat Sequences (PAWS), and we hereafter refer to this effort as DAWGPAWS.

\section{Implementation}

The DAWGPAWS workflow (Figure 1) is distributed as a suite of individual command line interface (CLI) programs written in the Perl programming language. Generally, each program is tailored for an individual step in the annotation process, and it can be used independently of all other programs in the package. This allows users to design an individualized annotation pipeline by selecting those computational components that are most appropriate to their annotation efforts. This modular design also facilitates using DAWGPAWS in a high throughput cluster-computing framework. Large-scale annotation jobs can be split across compute nodes by contigs being annotated as well as by the computational process used to generate computational results.

A common thread to each component of the DAWGPAWS package is that computational evidences are translated from the native annotation program output into the standard general feature format (GFF) [34]. The GFF file format facilitates integration of multiple computational results. This format can be directly curated by any biologist using standard sequence curation and visualization 


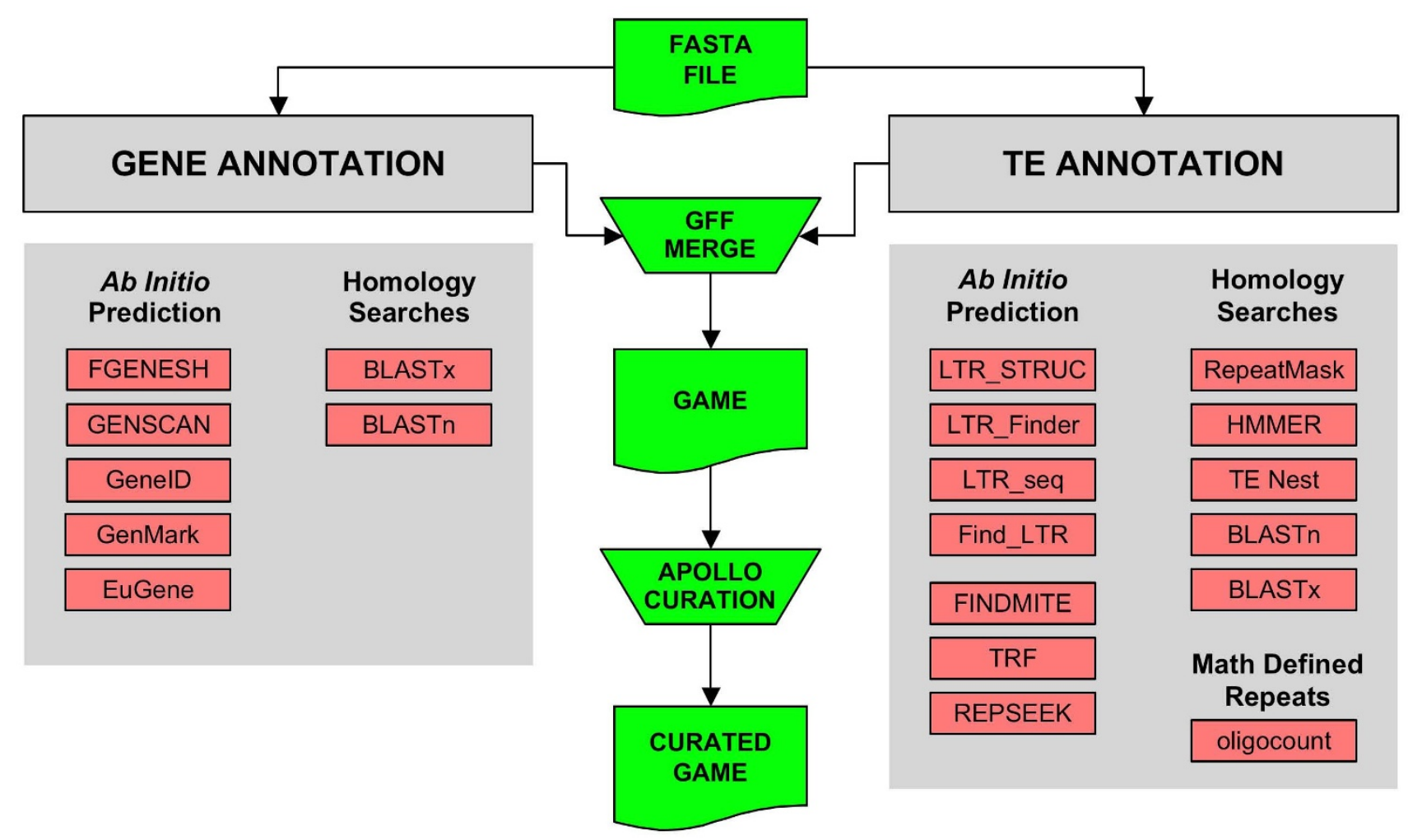

Figure I

An overview of the workflow supported by the current version of the DAWGPAWS suite of programs.

tools such as Apollo [35], Artemis [36], GBrowse [37], the UCSC genome browser [38] or the Ensembl Genome Browser [39]. The GFF files also provide a standard format for loading annotation results to relational database schemas such as BioSQL [40] or CHADO [41].

One of the main sets of scripts in the DAWGPAWS package is the batch run program set (Table 1). All of these scripts are designed to run individual annotation programs in a high throughput batch mode. They take as their input a directory of sequence files that are to be annotated and a configuration file describing the sets of parameters to use for each sequence file. The output of these batch scripts includes the original output from the annotation program as well as this output translated to the GFF format. The resulting files are stored in a predefined directory structure that allows users to quickly locate the original annotation results as well as the GFF copy. These batch programs exist for both gene and TE annotation results. The $a b$ initio gene annotation programs supported by these scripts include EuGène [9], GeneID [42], GeneMark.hmm [43], and Genscan [44]. The ab initio TE annotation programs that can be run in batch mode are
Find_LTR [45], LTR_STRUC [20], LTR_FINDER [21], LTR_seq [46], FINDMITE [19], and Tandem Repeats Finder [47]. Batch mode scripts also support TE annotation using HMMER [48], NCBI-BLAST [49], RepeatMasker [22], and TEnest [24]. The full set of gene and TE annotation programs that can be run in batch mode are summarized in Table 1.

In addition to the batch run programs, scripts that convert an individual annotation program output to GFF are also available (Table 2). These programs allow an existing annotation result to be specified, or they can take advantage of UNIX standard streams. If an input file is not specified, the conversion scripts will expect input from the standard input stream. Likewise, if the output path is not specified, these programs will write the output to a standard output stream. Accepting standard input and output streams facilitates using these programs as supplements to an existing workflow. For example, data can be piped directly from the output stream of an annotation program to a DAWGPAWS converter, and then piped on to a parser that loads the GFF formatted result to a database. These conversion programs provide the ability to support con- 
Table I: DAWGPAWS annotation scripts for generating computational annotation results in batch mode.

\begin{tabular}{|c|c|c|}
\hline Annotation Program & Result Type & DAWGPAWS Script \\
\hline EuGène [9] & Gene $a b$ initio and automated combined evidence & batch_eugene.pl \\
\hline GenelD [42] & Gene $a b$ initio & batch_geneid.pl \\
\hline GeneMark.hmm [43] & Gene $a b$ initio & batch_genemark.pl \\
\hline Genscan [44] & Gene $a b$ initio & batch_genescan.pl \\
\hline Find_LTR [45] & TE $a b$ initio & batch_findltr.pl* \\
\hline LTR_STRUC [20] & TE $a b$ initio & batch_Itrstruc.vbs \\
\hline LTR_FINDER [2I] & TE $a b$ initio & batch_Itrfinder.p/* \\
\hline LTR_seq [46] & TE $a b$ initio & batch_Itrseq.p|* \\
\hline FINDMITE [19] & TE $a b$ initio & batch_findmite.p $\left.\right|^{*}$ \\
\hline Tandem Repeats Finder [47] & Repeat $a b$ initio & batch_trf.pl \\
\hline HMMER [48] & TE homology & batch_hmmer.pl* \\
\hline NCBI-BLAST [49] & TE and gene homology & batch_blast.pl* \\
\hline RepeatMasker [22] & TE homology & batch_repmask.p|* \\
\hline TEnest [24] & TE homology & batch_tenest.pl \\
\hline
\end{tabular}

These scripts operate on a directory of FASTA files, and generate the native results of the annotation program as well as the GFF file format. The exception is the batch_Itrstruc.vbs visual basic script that must be used in conjunction with cnv_Itrstruc2gff.pl to generate results in GFF.

* Indicates programs that make use of a configuration file. The nature and format of the configuration file for these programs is described in the individual help file for those programs.

version of output from programs such as FGENESH [50,51] and RepSeek [52] that are not supported by batch scripts in DAWGPAWS.

The DAWGPAWS suite also includes specialized tools for TE annotation. For identification of the highly repetitive regions of a contig, the seq_oligocount.pl program can count the occurrence of oligomers in the query sequence against an index of random shotgun sequences. This program generates all oligomers of length $k$ from the query sequence, and uses the vmatch program [53] to determine the number of these k-mers that occur in a random shotgun sequence data set generated by mkvtree [53]. The output of this program is a GFF file indicating the count of these k-mers in the shotgun sequence dataset. These results may be used to identify the mathematically defined repeats in the query sequence, as well as provides a means to visualize low-copy-number runs in the query sequence [54].
In addition to the gene and TE annotation-specific scripts included in the DAWGPAWS package, helper applications are also included (Table 3 ). These CLI programs fulfill needs that occur when generating annotation results. They allow for file conversion such as the conversion of GFF to game.xml format or the conversion of a lowercase masked sequence file to a hard masked sequence file. They also prepare the sequence files for annotation by shortening FASTA headers as required by some programs, or by splitting a single FASTA file containing multiple records into multiple FASTA files containing single record files. The ability to generate Euler Diagrams is also supported via the vennseq.pl conversion script that formats GFF file data for input into the VennMaster program [55].

A CLI interface was selected for DAWGPAWS to facilitate the use of our applications in a cluster-computing environment, and to provide stability in program interface across multiple operating systems. While command line 
Table 2: DAWGPAWS scripts for conversion of annotation results from native program output to GFF.

\begin{tabular}{lll}
\hline Annotation Program & Result Type & DAWGPAWS Script \\
\hline FGENESH [50,5I] & Gene $a b$ initio & cnv_fgenesh2gff.pl \\
\hline GeneMark.hmm [43] & Gene $a b$ initio & cnv_genemark2gff.pl \\
\hline Find_LTR [45] & TE ab initio & cnv_findltr2gff.pl \\
\hline LTR_FINDER [21] & TE ab initio & cnv_ltrfinder2gff.pl \\
\hline LTR_seq [46] & TE ab initio & cnv_Itrseq2gff.pl \\
\hline LTR_STRUC [20] & TE ab initio & cnv_Itrstruc2gff.pl \\
\hline RepSeek [52] & TE ab initio & cnv_repseek2gff.pl \\
\hline NCBI-BLAST [49] & TE and gene homology & cnv_blast2gff.pl \\
\hline RepeatMasker [22] & TE homology & cnv_repmask2gff.pl \\
\hline TEnest [24] & TE homology & cnv_tenest2gff.pl
\end{tabular}

interface programs may be daunting to some users, every effort has been made to simplify their use. All of the CLI programs included in the DAWGPAWS suite follow consistent protocols for command line options (Table 4). Help files or full program manuals are available from the command line within all programs by invoking the - help or - man options. These application manuals are also available in HTML form on the DAWGPAWS website along with a general program manual describing the installation and use of a local implementation of the DAWGPAWS package [56]. This documentation is also included in the downloadable release of DAWGPAWS.

\section{Results and discussion}

The computational annotation results generated by DAWGPAWS can be directly imported into any genome annotation program that supports GFF. We have used the Apollo program [35] to visualize and curate our results for genes and transposable elements in the wheat genome (Figure 2). Since the game $\mathrm{xml}$ file format is the most stable way to store annotation results in Apollo, it is generally useful to first convert GFF files to the game xml format before beginning curation of computational results. The visual display of computational results in Apollo is modified by a tiers configuration file. This file controls how and where individual computational and annotation results are drawn on the annotation pane. The tiers file used in these annotation efforts is included in the DAWGPAWS download package, and it can serve as a starting point for generating individualized tier files for other plant annotation efforts. As an alternative to Apollo, it is also possible to curate computational results using the Artemis sequence visualization program [36].

The GBrowse package [37] can also visualize GFF formatted annotations, and has proven to be a useful method for visualizing TE results. GBrowse makes use of core images called glyphs that are used to draw sequence features along a genome. The available glyphs in GBrowse can be supplemented by writing additional Perl modules, and we have generated TE glyphs that allow visualization of the biologically relevant features of TEs. GBrowse also has the capability to draw histograms along the sequence contigs. GBrowse can thus combine TE glyphs and histograms to provide an informative visualization of the distribution of mathematically defined repeats and the structural features of TEs (Figure 3). The current drawback to visualizations in GBrowse is that the program is intended to serve as a static visualization tool, and does not provide the means for the curation and combination of computational results. It would therefore be helpful if the current curation programs for gene annotation, such as Apollo or Artemis, directly addressed the needs of TE annotation curation and developed glyphs for the major classes of TEs.

In addition to visualization and curation of the annotated DNA, it is also possible to transfer the DAWGPAWS results into existing database schema. For example, the CHADO database [41] can make use of the gmod_bulk_load_gff3.pl program [57] that can load GFF3 format files into a CHADO database. In the DAWG- 
Table 3: Additional helper scripts included in the DAWGPAWS package.

\begin{tabular}{|c|c|}
\hline DAWGPAWS Script & Purpose \\
\hline cnv_gff2game.pl & Converts GFF files to the game.xml format. \\
\hline cnv_game2gff3.pl & Converts game.xml files to the GFF3 format. \\
\hline batch_hardmask.pl & $\begin{array}{l}\text { Given a directory of lowercase masked sequence files, this will replace lowercase residues with an } \mathrm{N} \text { or } \mathrm{X} \text { to indicate } \\
\text { masking. }\end{array}$ \\
\hline dir_merge.pl & $\begin{array}{l}\text { Given annotation results scattered across multiple directories, this program can merge the results into subdirectories in } \\
\text { a single parent directory. }\end{array}$ \\
\hline vennseq.pl & $\begin{array}{l}\text { Given GFF annotation results from multiple methods, this program generates a Euler Diagram of these features using the } \\
\text { VennMaster program [55] }\end{array}$ \\
\hline batch_findgaps.pl & This program will annotate gaps in the query sequences in the input directory. \\
\hline clust_write_shell.pl & $\begin{array}{l}\text { This program writes shell scripts to run DAWGPAWS in a cluster environment running the Platform LSF queuing } \\
\text { system. }\end{array}$ \\
\hline cnv_seq2dir.pl & $\begin{array}{l}\text { Given a FASTA file with multiple sequence files, this program generates a separate FASTA file for each sequence record. } \\
\text { The sequence files produced are named using the sequence ID in the FASTA header in the input file. }\end{array}$ \\
\hline fasta_merge.pl & This program merges all FASTA files in a directory into a single FASTA file. \\
\hline fasta_shorten.pl & $\begin{array}{l}\text { This program shortens the FASTA header by limiting the header length, or splitting the header by a delimiting character. } \\
\text { Some annotation programs are limited by the length of the FASTA header that is accepted, and this programs allows } \\
\text { input files to meet this limitation. }\end{array}$ \\
\hline fetch_tenest.pl & Fetches multiple results from the Plant GDB TEnest server and converts the results to GFF. \\
\hline gff_seg.pl & $\begin{array}{l}\text { Given a GFF file that contains point or segment data, this will extract segments with score values that exceed a threshold } \\
\text { value. }\end{array}$ \\
\hline Itrstruc_prep.pl & $\begin{array}{l}\text { Because the LTR_STRUC program only runs under the windows environment, this program converts FASTA sequences } \\
\text { in UNIX to DOS line endings and generates the files name and flist file required for LTR_STRUC. }\end{array}$ \\
\hline seq_oligiocount.pl & $\begin{array}{l}\text { This program allows for the generation of a GFF file that counts the number of times an oligomer in the genomic contig } \\
\text { occurs in a reference shotgun sequence database. }\end{array}$ \\
\hline
\end{tabular}

PAWS package, the GFF3 format files from curated results can be generated with the cnv_game2gff3.pl program. These curated results could then be stored in a local implementation of the CHADO database. The BioSQL database schema [40] also includes a bp_load_gff.pl script that can load GFF results into the database schema.

The DAWGPAWS annotation framework has a number of features that make it a good choice to facilitate the workflow in plant genome annotation. The use of configuration files makes it fairly easy to modify the annotation workflow for the species of interest. The configuration files also makes it quite easy to generate results with multiple parameter sets for an individual program. Using multiple parameter sets will be especially useful when working with a genome that has not been annotated before, and for which appropriate annotation parameters have not been identified. Also, while previous annotation pipelines have focused on gene annotation, the DAWGPAWS suite maximizes the quality of TE annotation results. Most plants contain genomes with sizes $>5000$ $\mathrm{Mb}$ [58], and are therefore expected to contain more than $80 \%$ TEs [59], so efficiently dealing with this large number and diverse set of mobile DNAs is necessary for effective genome annotation.

The current focus of DAWGPAWS in our laboratory is the structural annotation of the genes and TEs in a genome using methods and applications tuned to the Triticeae. In annotation of 220 BACs from hexaploid bread wheat, we found that the DAWGPAWS pipeline increased the rate of individual BAC annotations by twenty-fold. Due to the 
Table 4: Common command line options used throughout the DAWGPAWS suite of programs.

\begin{tabular}{|c|c|}
\hline Option & Description \\
\hline $\begin{array}{l}\text {--indir or } \\
\text {--infile }\end{array}$ & $\begin{array}{l}\text { For batch scripts, this indicates the input directory containing the FASTA files to annotate. For conversion scripts, this indicates the } \\
\text { input file to convert from the native format to the GFF format. }\end{array}$ \\
\hline $\begin{array}{l}\text {--outdir or } \\
\text {--outfile }\end{array}$ & $\begin{array}{l}\text { For batch scripts, this indicates the output directory containing the annotation results for the program and the GFF results. } \\
\text { For conversion scripts, this indicates the path to the GFF output file. }\end{array}$ \\
\hline --config & For programs that make use of a configuration file, this indicates the path to the configuration file to use. \\
\hline --seqname & For conversion scripts, this indicates the sequence id to use in the GFF output file. \\
\hline --param & $\begin{array}{l}\text { For conversion scripts, this indicates the name of that parameter set used with the annotation program. This option allows the user } \\
\text { to distinguish among multiple parameter sets for the same annotation program, and this parameter name is appended to the source } \\
\text { column of the GFF output file. }\end{array}$ \\
\hline --program & For conversion scripts, this indicates the name of the program used to generate the annotation result. \\
\hline --version & Print the current version of the script. \\
\hline --usage & Print a short program usage message. \\
\hline --help & Print a short help message including the common usage and all program options available at the command line. \\
\hline$--\operatorname{man}$ & Print the full program manual. \\
\hline --verbose & $\begin{array}{l}\text { This will run the program with maximum verbosity. This option will generate status updates while the program is running, and will } \\
\text { maximize the error reporting functions of the script. All verbose statements are written to the standard error output stream. }\end{array}$ \\
\hline
\end{tabular}

time required to manually generate annotation results, this previous annotation effort was limited to using the FGENESH annotation program combined with a BLAST search of predicted models against known transposable elements and protein databases [60]. Using this method, annotators could annotate a single BAC in one to two days. The implementation of the DAWGPAWS pipeline increased the speed of annotation to ten-fifteen BACs per person per day. Furthermore, the quality of both TE and gene prediction were also seen to improve with the use of DAWGPAWS. This was due, at least in part, to the larger number of complementary programs for TE and gene discovery that could be conveniently employed in each BAC annotation. Specifically, the inclusion of $a b$ initio TE prediction programs allowed for the identification of new families of LTR retrotransposons that would have been missed in our previous annotation efforts. Predicted gene models that span these newly discovered families would not have been identified as TEs in the exclusively homology-based searches that were previously used.

Future development of DAWGPAWS will incorporate tools for the functional annotation of the predicted genes. Currently, functional annotation can be done within the Apollo program by manually selecting individual gene models and BLASTing these results against appropriate databases. A batch run support for additional local alignment search tools will also be added. The use of NCBIBLAST is sufficient for most comparisons of sequence contigs against reference databases, but programs such as BLAT [61] or sim 4 [62] are designed specifically to align ESTs and flcDNAs against assembled genomes. While output from these local alignment tools can be converted to GFF using the existing cnv_blast2gff.pl program in DAWGPAWS, it would be useful to use these packages in a batch run framework similar to the batch_blast.pl program.

Support for additional $a b$ initio gene annotation programs will also be added to future releases of DAWGPAWS. Augustus [63] is an $a b$ initio annotation program that will be useful for gene annotation that seeks to identify all transcripts derived from a single locus. Support for GENEZILLA [64] and GlimmerHMM [64] gene annotation packages will also be added to future releases of DAWGPAWS. The SNAP program [65] will be added to support the annotation of genomes that have been sequenced $d e$ novo and lack species-specific HMM model parameterizations. The addition of the PASA [66] program would assist in the annotation of genomes that have large transcript databases that can assist genome annotation. As additional fully-sequenced genomes are added to the plant 


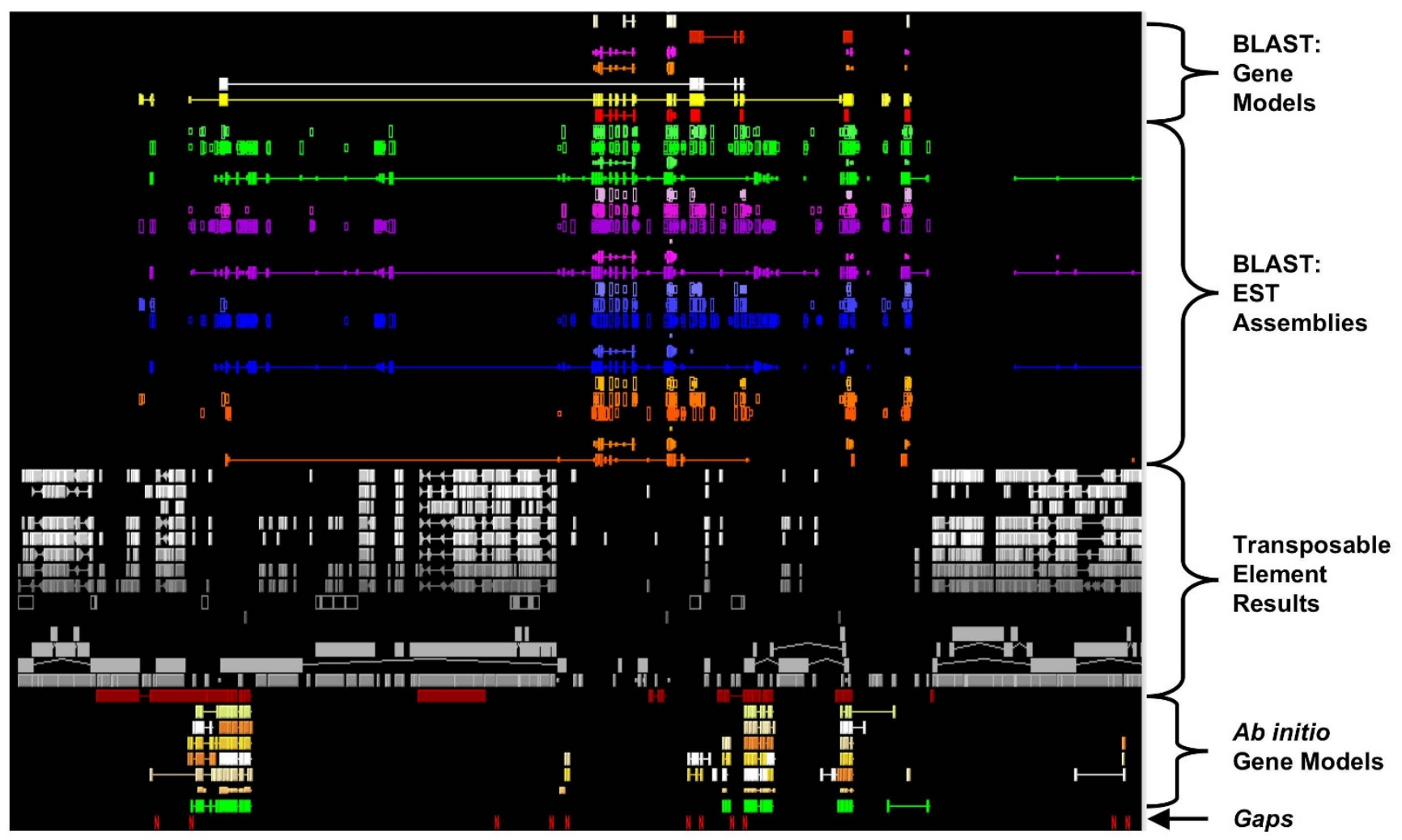

Figure 2

Screen capture image of gene and TE annotation results visualized in the Apollo genome annotation program. This example shown is for a wheat BAC that has been annotated and curated with the assistance of DAWGPAWS.

genomics literature, we can make use of syntenic comparisons and multiple alignments to aid in gene annotation [67] as well as TE annotation [68]. Future development of DAWGPAWS will incorporate syntenic alignment and prediction programs such as SGP2 [69], SLAM [70], and TWINSCAN [71] as they become increasingly relevant to plant genome annotation.

\section{Conclusion}

The DAWGPAWS annotation workflow provides a suite of command line interface programs that can generate computational evidences for human curation in a highthroughput fashion. We have used the DAWGPAWS pipeline to annotate 220 randomly selected BACs with wheat DNA inserts for both gene and TE content. Our curation efforts on the DAWGPAWS output are implemented in the Apollo program. The tiers file used for visualization of this curation are available as part of the DAWGPAWS package.

DAWGPAWS represents an efficient tool for genome annotation in the Triticeae, and can be used in its current form to generate gene and TE computational results for other grass genomes. Minor modifications to the configu- ration files used by DAWGPAWS can make this program suitable to the generation of computational annotation results for any plant genome. The TE annotation capabilities of DAWGPAWS exceeds any other current genome annotation suite, and makes this package particularly valuable for the great majority of plant genomes, such as wheat or maize, that contain a diverse arrays of TEs that comprise the majority of the nuclear genome.

The DAWGPAWS program has been specifically designed to facilitate use of individual component scripts outside of the entire package. Each script can function independently of all other applications in the package, and programs make use of standard input and standard output streams when possible to facilitate integration into existing pipelines. Since this package is being released under the open source GPL (version 3), the suite and its individual components can be used and modified under the terms of the GPL. Template batch run and conversion scripts are provided in a boilerplate format to facilitate extending DAWGPAWS to additional annotation tools. Furthermore, since we have selected the Perl language for the implementation of our package, the addition of new annotation tools can leverage existing modules in the 


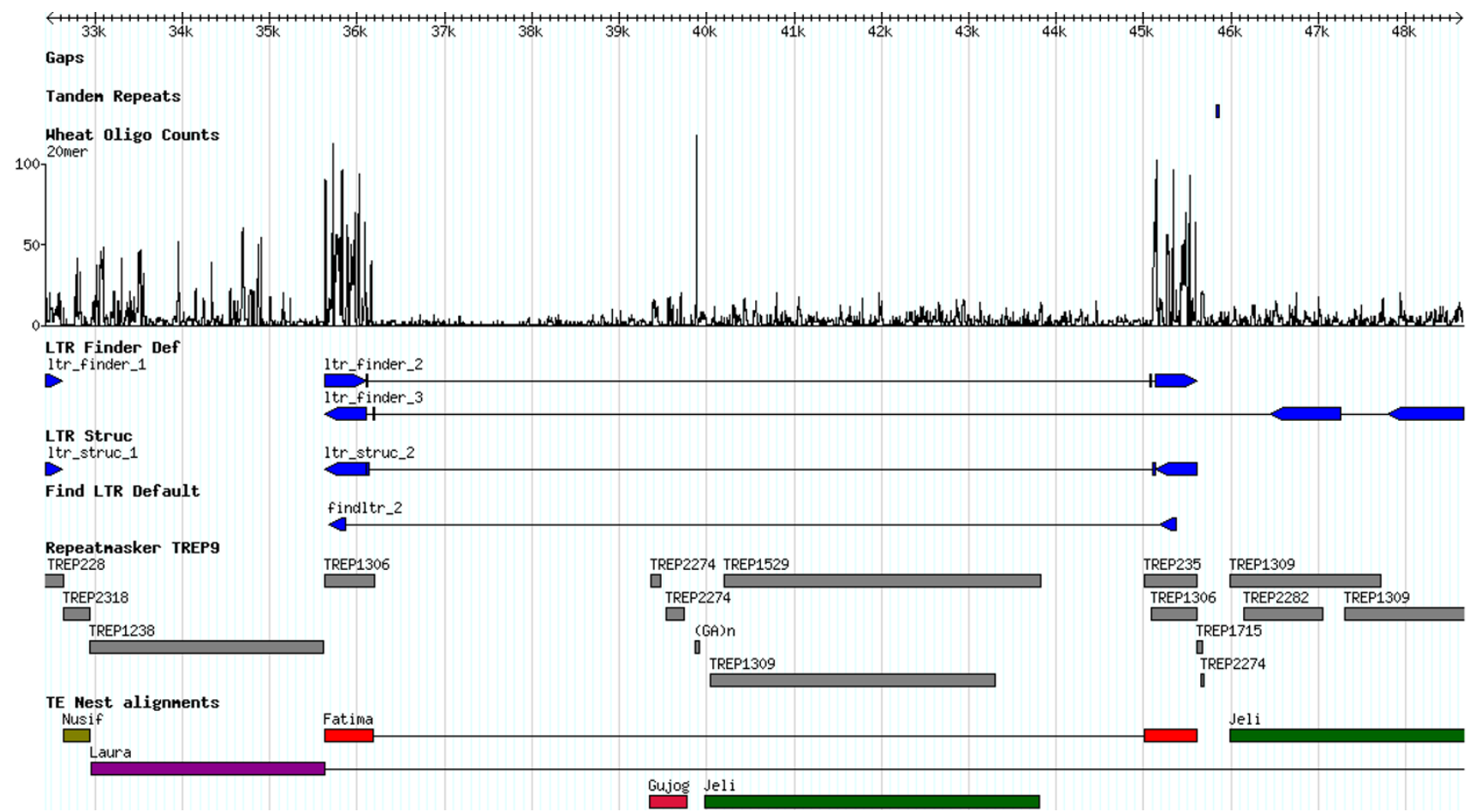

Figure 3

Screen capture image of the TE annotation results and oligomer counts visualized in the GBrowse genome annotation visualization program. The example shown is for a $I 5 \mathrm{~kb}$ segment of a BAC with a wheat DNA insert.

BioPerl toolkit [72]. These modules include parsers for computational tools useful for predicting alternative splicing $[62,61]$ as well as interfaces for transfer RNA prediction [73]. We also formally invite collaboration in the development of additional DAWGPAWS applications under the auspices of the GNU GPL, as facilitated by the SourceForge subversion repository of the DAWGPAWS source code. Interested collaborators may contact the authors or become member developers of the DAWGPAWS SourceForge project [74].

\section{Availability and requirements}

Project Name: DAWGPAWS Plant Genome Annotation Pipeline

Project Home Page: http://dawgpaws.sourceforge.net/

Operating System: Platform Independent

Programming Language: Perl

Other Requirements: BioPerl 1.4, as well as the annotation programs that scripts are dependent upon.

License: GNU General Public License 3
Any restrictions to use by non-academics: No restrictions

\section{Abbreviations}

BAC: Bacterial Artificial Chromosome; cDNA: complementary DNA; CLI: Command Line Interface; EST: Expressed Sequence Tag; flcDNA: full-length complementary DNA; GFF: General Feature Format; GPL: General Public License; HMM: Hidden Markov Model; LTR: Long Terminal Repeat; ORF: Open Reading Frame; pHMM: Profile Hidden Markov Model; TE: Transposable Element

\section{Competing interests}

The authors declare that they have no competing interests.

\section{Authors' contributions}

JE developed the pipeline, wrote the software, and drafted the manuscript. JB conceived the study, oversaw pipeline development, and helped draft the manuscript. All authors read and approved the final manuscript.

\section{Acknowledgements}

The authors would like to thank Katrien Devos, Antonio Costa de Oliveira, Xiangyang $\mathrm{Xu}$, Ansuya Jogi, and Jennifer Hawkins for their useful feedback that has been incorporated in the implementation of DAWGPAWS.

Xiangyang Xu provided helpful comments on a draft version of this manuscript. The submitted version of this manuscript was refined with construc- 
tive comments from two anonymous peer reviewers. This work was supported by NSF grants DBI-050I8I4 and DBI-0607/23.

\section{References}

I. Liolios K, Mavromatis K, Tavernarakis N, Kyrpides NC: The Genomes On Line Database (GOLD) in 2007: status of genomic and metagenomic projects and their associated metadata. Nucleic Acids Res 2008, 36:D475-479.

2. Wheeler DL, Barrett T, Benson DA, Bryant SH, Canese K, Chetvernin V, Church DM, Dicuccio M, Edgar R, Federhen S, et al.: Database resources of the National Center for Biotechnology Information. Nucleic Acids Res 2008, 36:DI3-2I.

3. Pop M, Salzberg SL: Bioinformatics challenges of new sequencing technology. Trends Genet 2008, 24:|42-| 49.

4. Stein L: Genome annotation: from sequence to biology. Nat Rev Genet 2001, 2:493-503.

5. Bennetzen JL: Transposable element contributions to plant gene and genome evolution. Plant Mol Biol 2000, 42:25 I-269.

6. Bennetzen JL: Transposable elements, gene creation and genome rearrangement in flowering plants. Curr Opin Genet Dev 2005, I 5:621-627

7. Wang Z, Chen Y, Li Y: A brief review of computational gene prediction methods. Genomics Proteomics Bioinformatics 2004, 2:216-22।.

8. Do JH, Choi DK: Computational approaches to gene prediction. J Microbiol 2006, 44: I37-I44.

9. Schiex T, Moisan A, Rouzé P: EuGene: An Eucaryotic Gene Finder that combines several sources of evidence. Computational Biology 200 I: I I I-I 25.

10. Allen JE, Salzberg SL: JIGSAW: integration of multiple sources of evidence for gene prediction. Bioinformatics 2005, 2 I:3596-3603

II. Bennetzen JL, Coleman C, Liu R, Ma J, Ramakrishna W: Consistent over-estimation of gene number in complex plant genomes. Curr Opin Plant Biol 2004, 7:732-736.

12. Sanmiguel $P$, Bennetzen JL: Evidence that a recent increase in maize genome size was caused by the massive amplification of intergene retrotransposons. Annals of Botany 1998, 82:37-44.

13. Bergman CM, Quesneville H: Discovering and detecting transposable elements in genome sequences. Brief Bioinform 2007, 8:382-392.

14. Feschotte C, Pritham EJ: Computational analysis and paleogenomics of interspersed repeats in eukaryotes. In Computational Genomics: Current Methods Stojanovic N: Taylor and Francis; 2007:3 I-54.

15. Saha S, Bridges S, Magbanua ZV, Peterson DG: Computational Approaches and Tools Used in Identification of Dispersed Repetitive DNA Sequences. Tropical Plant Biology 2008, I:85-96.

16. Bao Z, Eddy SR: Automated de novo identification of repeat sequence families in sequenced genomes. Genome Res 2002, I 2: I269- I 276 .

17. Edgar RC, Myers EW: PILER: identification and classification of genomic repeats. Bioinformatics 2005, 2 I (SuppI I):i I 52-I58.

18. Price AL, Jones NC, Pevzner PA: De novo identification of repeat families in large genomes. Bioinformatics 2005, 2 I(Suppl I):i35I-358.

19. Tu Z: Eight novel families of miniature inverted repeat transposable elements in the African malaria mosquito, Anopheles gambiae. Proc Natl Acad Sci USA 200I, 98: I699-I704.

20. McCarthy EM, McDonald JF: LTR STRUC: a novel search and identification program for LTR retrotransposons. Bioinformatics 2003, 19:362-367.

21. Xu Z, Wang H: LTR FINDER: an efficient tool for the prediction of full-length LTR retrotransposons. Nucleic Acids Res 2007, 35:W265-268.

22. Smit A, Hubley R, Green P: RepeatMasker Open-3.0. I 996-2004. [http://www.repeatmasker.org/cgi-bin/WEBRepeatMasker].

23. Kohany O, Gentles AJ, Hankus L, Jurka J: Annotation, submission and screening of repetitive elements in Repbase: RepbaseSubmitter and Censor. BMC Bioinformatics 2006, 7:474.

24. Kronmiller BA, Wise RP: TEnest: Automated chronological annotation and visualization of nested plant transposable elements. Plant Physiol 2008, I 46:45-59.

25. Pereira V: Automated paleontology of repetitive DNA with REANNOTATE. BMC Genomics 2008, 9:614.
26. Wicker T, Matthews DE, Keller B: TREP: a database for Triticeae repetitive elements. Trends in Plant Science 2002, 7:561-562.

27. Ouyang S, Buell CR: The TIGR Plant Repeat Databases: a collective resource for the identification of repetitive sequences in plants. Nucleic Acids Res 2004, 32:D360-363.

28. Jurka J, Kapitonov VV, Pavlicek A, Klonowski P, Kohany O, Walichiewicz J: Repbase Update, a database of eukaryotic repetitive elements. Cytogenet Genome Res 2005, I I 0:462-467.

29. Spannagl M, Noubibou O, Haase D, Yang L, Gundlach H, Hindemitt T, Klee K, Haberer G, Schoof H, Mayer KF: MIPSPIantsDB - plant database resource for integrative and comparative plant genome research. Nucleic Acids Res 2007, 35:D834-840.

30. Li R, Ye J, Li S, Wang J, Han Y, Ye C, Yang H, Yu J, Wong GK: ReAS: Recovery of ancestral sequences for transposable elements from the unassembled reads of a whole genome shotgun. PLoS Comput Biol 2005, I:e43.

31. DeBarry JD, Liu R, Bennetzen JL: Discovery and assembly of repeat family pseudomolecules from sparse genomic sequence data using the Assisted Automated Assembler of Repeat Families (AAARF) algorithm. BMC Bioinformatics 2008, 9:235.

32. Kurtz S, Narechania A, Stein JC, Ware D: A new method to compute K-mer frequencies and its application to annotate large repetitive plant genomes. BMC Genomics 2008, 9:5 I7.

33. Quesneville H, Bergman CM, Andrieu O, Autard D, Nouaud D, Ashburner M, Anxolabehere D: Combined evidence annotation of transposable elements in genome sequences. PLoS Comput Biol 2005, I: I66-175.

34. GFF Format Specifications [http://www.sanger.ac.uk/Software/ formats/GFF/GFF Spec.shtml]

35. Lewis SE, Searle SM, Harris N, Gibson M, Lyer V, Richter J, Wiel C, Bayraktaroglir L, Birney E, Crosby MA, et al.: Apollo: a sequence annotation editor. Genome Biol 2002, 3:RESEARCH0082.

36. Rutherford K, Parkhill J, Crook J, Horsnell T, Rice P, Rajandream MA Barrell B: Artemis: sequence visualization and annotation. Bioinformatics 2000, 16:944-945.

37. Donlin MJ: Using the Generic Genome Browser (GBrowse). Curr Protoc Bioinformatics 2007, Chapter 9(Unit 9):9.

38. Kuhn RM, Karolchik D, Zweig AS, Wang T, Smith KE, Rosenbloom KR, Rhead B, Raney BJ, Pohl A, Pheasant M, et al:: The UCSC Genome Browser Database: update 2009. Nucleic Acids Res 2009, 37:D755-76I.

39. Stalker J, Gibbins B, Meidl P, Smith J, Spooner W, Hotz HR, Cox AV: The Ensembl Web site: mechanics of a genome browser. Genome Res 2004, 14:951-955.

40. BioSQL [http://www.biosql.org]

41. Zhou P, Emmert D, Zhang P: Using Chado to store genome annotation data. Curr Protoc Bioinformatics 2006, Chapter 9(Unit 9):6.

42. Parra G, Blanco E, Guigo R: GenelD in Drosophila. Genome Res 2000, 10:5II-5I5.

43. Lukashin AV, Borodovsky M: GeneMark.hmm: new solutions for gene finding. Nucleic Acids Res 1998, 26: I 107-I I I 5.

44. Burge C, Karlin S: Prediction of complete gene structures in human genomic DNA. J Mol Biol 1997, 268:78-94.

45. Rho M, Choi JH, Kim S, Lynch M, Tang H: De novo identification of LTR retrotransposons in eukaryotic genomes. BMC Genomics 2007, 8:90.

46. Kalyanaraman A, Aluru S: Efficient algorithms and software for detection of full-length LTR retrotransposons. J Bioinform Comput Biol 2006, 4:197-216.

47. Benson G: Tandem repeats finder: a program to analyze DNA sequences. Nucleic Acids Res 1999, 27:573-580.

48. Eddy SR: Profile hidden Markov models. Bioinformatics 1998 , I 4:755-763.

49. Altschul SF, Gish W, Miller W, Myers EW, Lipman DJ: Basic local alignment search tool. J Mol Biol 1990, 2 I 5:403-4I0.

50. Solovyev VV, Salamov AA, Lawrence CB: Identification of human gene structure using linear discriminant functions and dynamic programming. Proc Int Conf Intell Syst Mol Biol 1995, 3:367-375.

51. Salamov AA, Solovyev VV: Ab initio gene finding in Drosophila genomic DNA. Genome Res 2000, 10:516-522.

52. Achaz G, Boyer F, Rocha EP, Viari A, Coissac E: Repseek, a tool to retrieve approximate repeats from large DNA sequences. Bioinformatics 2007, 23:119-121. 
53. vmatch [http://www.vmatch.de/]

54. Wicker T, Narechania A, Sabot F, Stein J, Vu GT, Graner A, Ware D, Stein N: Low-pass shotgun sequencing of the barley genome facilitates rapid identification of genes, conserved non-coding sequences and novel repeats. BMC Genomics 2008, 9:5 I8.

55. Kestler HA, Muller A, Gress TM, Buchholz M: Generalized Venn diagrams: a new method of visualizing complex genetic set relations. Bioinformatics 2005, 21:1592-1595.

56. DAWGPAWS User Manual [http://dawgpaws.sourceforge.net/ man.html]

57. How to Load GFF Into Chado [http://gmod.org/wiki/ Load GFF Into Chado]

58. Zonneveld BJM, Leitch IJ, Bennett MD: First nuclear DNA amounts in more than $\mathbf{3 0 0}$ angiosperms. Annals of Botany 2005, 96:229-244.

59. Flavell RB, Bennett MD, Smith JB, Smith DB: Genome Size and Proportion of Repeated Nucleotide-Sequence DNA in Plants. Biochemical Genetics 1974, I 2:257-269.

60. Devos KM, Costa de Oliveira A, Xu X, Estill JC, Estep M, Jogi A, Morales M, Pinheiro J, SanMiguel P, Bennetzen JL: Structure and organization of the wheat genome - the number of genes in the hexaploid wheat genome. II th International Wheat Genetics Symposium 2008 Proceedings 2008: I-5 [http://ses.library.usyd.edu.aul bitstream/2123/3389/1/O25.pdf]. Sydney University Press

61. Kent WJ: BLAT - the BLAST-like alignment tool. Genome Res 2002, I 2:656-664

62. Florea L, Hartzell G, Zhang Z, Rubin GM, Miller W: A computer program for aligning a cDNA sequence with a genomic DNA sequence. Genome Res 1998, 8:967-974.

63. Stanke M, Keller O, Gunduz I, Hayes A, Waack S, Morgenstern B: AUGUSTUS: ab initio prediction of alternative transcripts. Nucleic Acids Res 2006, 34:W435-439.

64. Majoros WH, Pertea M, Salzberg SL: TigrScan and GlimmerHMM: two open source ab initio eukaryotic gene-finders. Bioinformatics 2004, 20:2878-2879.

65. Korf I: Gene finding in novel genomes. BMC Bioinformatics 2004, 5:59.

66. Haas BJ, Delcher AL, Mount SM, Wortman JR, Smith RK Jr, Hannick LI, Maiti R, Ronning CM, Rusch DB, Town CD, et al:: Improving the Arabidopsis genome annotation using maximal transcript alignment assemblies. Nucleic Acids Res 2003, 31:5654-5666.

67. Dubchak I: Comparative analysis and visualization of genomic sequences using VISTA browser and associated computational tools. Methods Mol Biol 2007, 395:3-16.

68. Caspi A, Pachter L: Identification of transposable elements using multiple alignments of related genomes. Genome Res 2006, 16:260-270.

69. Parra G, Agarwal P, Abril JF, Wiehe T, Fickett JW, Guigo R: Comparative gene prediction in human and mouse. Genome Res 2003, 13:108-117.

70. Alexandersson M, Cawley S, Pachter L: SLAM: cross-species gene finding and alignment with a generalized pair hidden Markov model. Genome Res 2003, 13:496-502.

7I. Korf I, Flicek P, Duan D, Brent MR: Integrating genomic homology into gene structure prediction. Bioinformatics 200I, I7(Suppl I):S|40-|48.

72. Stajich JE, Block D, Boulez K, Brenner SE, Chervitz SA, Dagdigian C, Fuellen G, Gilbert JG, Korf I, Lapp H, et al:: The Bioperl toolkit: Perl modules for the life sciences. Genome Res 2002, 12:1611-1618

73. Lowe TM, Eddy SR: tRNAscan-SE: a program for improved detection of transfer RNA genes in genomic sequence. Nucleic Acids Res 1997, 25:955-964.

74. DAWGPAWS SourceForge Project Page [http://source forge.net/projects/dawgpaws/]
Publish with Bio Med Central and every scientist can read your work free of charge

"BioMed Central will be the most significant development for disseminating the results of biomedical research in our lifetime. "

Sir Paul Nurse, Cancer Research UK

Your research papers will be:

- available free of charge to the entire biomedical community

- peer reviewed and published immediately upon acceptance

- cited in PubMed and archived on PubMed Central

- yours - you keep the copyright

Submit your manuscript here:

http://www.biomedcentral.com/info/publishing_adv.asp
BiolMedcentral 